\title{
Some Aspects of Turbulence in Discrete Mechanics
}

\section{Quelques aspects de la turbulence en mécanique discrète}

\author{
Jean-Paul Caltagirone ${ }^{1}$, Stéphane Vincent ${ }^{2}$ \\ ${ }^{1}$ Université de Bordeaux, Institut de Mécanique et d'Ingéniérie, Département TREFLE, UMR CNRS n 5295, 16 Avenue \\ Pey-Berland, 33607 Pessac Cedex, calta @ipb.fr \\ ${ }^{2}$ Université Paris-Est Marne-La-Vallée, Laboratoire Modélisation et Simulation Multi Echelle (MSME), UMR CNRS n 8208 , \\ 5 boulevard Descartes, 77454 Marne-la-Vallée Cedex, stephane.vincent@u-pem.fr
}

\begin{abstract}
The discrete mechanics formalism and equations are considered in the present work in order to establish the role played by representative motion equations on the study of turbulence in fluids. In particular, a set of differences related to the turbulent pressure, the dynamics of vorticity in two spatial dimensions, the turbulent dissipation or the divergence of acceleration are discussed compared to the classical continuous media and Navier-Stokes equations. A second part is devoted to presenting on a first example, the rigid rotational motion, the differences between discrete and continuum mechanics. A last section is devoted to simulating the turbulent channel flow at turbulent Reynolds number of $R e_{\tau}=590$. It is demonstrated that discrete mechanics allow to recover accurately the mean velocity profiles of reference DNS and also to provide scale laws of the whole mean velocity profile from the wall to the center of the channel.

KEYWORDS. Discrete Mechanics, Hodge-Helmholtz decomposition, Lamb vector,Turbulent channel flow, Law of the wall, Dynamics of the vorticity, turbulent dissipation.
\end{abstract}

\section{Introduction}

Starting from the first drawings of Da Vinci [11], when one is interested in turbulence and its understanding, it can be chosen to adopt different complementary scientific approaches that have historically been experimental [16], theoretical [18, 7] or numerical [2]. In the framework of this work, we choose the prism of the formulation of the models in fluid mechanics to propose a certain number of remarks and discussions related to turbulence and the phenomena it is sensitive to. If we rely here on the importance and meaning of models in the analysis and understanding of turbulence, this is particularly because new formulations of fluid mechanics equations have been published in recent years, more specifically those associated to Discrete Mechanics (DM) [1].

On a general point of view, the notion of discrete medium is directly derived from the principle of relativity of velocity and weak equivalence principle: gravity accelerates all objects regardless of their masses or the materials from which they are made. All the contributions brought by the mechanical effects such as viscosity, compression or inertia as well as all the source terms, are written as the sum of a free divergence term and a zero rotational term following the Hodge-Helmholtz decomposition. The motion equations of discrete mechanics reveals the role played by two quantities, namely the scalar and vector potentials, both associated with acceleration, a quantity considered as absolute. Even if the results obtained with this set of equations are generally the same as those of the continuous media provided by the Navier-Stokes equations, many formal differences exist. In particular, the density, as such, has disappeared from the momentum equation in favor of a scalar potential being the ratio between pressure and density. Many properties of the continuum are recovered intrinsically by the discrete mechanics formalism, in particular the conservation of mass, rotational or kinetic energy to cite a few. Trying to make the link between discrete or continuum mechanics formulations and analyzing or understanding of turbulence appears to us as a relevant issue to be tackled with. 
Based on formulation differences, some features of discrete mechanics directly impact the understanding and modeling of turbulence. One of them relates to the value of the compressive viscosity for fluids, which is classically fixed by Stokes' law and is clearly questioned. The treatment of the pressure in direct numerical simulation or the turbulent pressure in statistical turbulence modeling is directly related to the value of this compression viscosity, especially at the time scales of the small turbulent structures. The notion of incompressibility is also closely related to the definition of a characteristic time and a direct simulation of turbulence can not underestimate this phenomenon, even in liquids. To quote another crucial aspect of turbulence generation and control, the inertial terms of the Navier-Stokes equation can be written in the form of a Lamb vector but this vector is not a rotational, which leads to the proven difference between 2D and 3D turbulence. In discrete mechanics, the Lamb vector does not exist in the formulation of the inertial term, which is replaced by the dual rotational of an inertial potential. Regarding shearing and energy transfer, we find a similar difficulty on the viscous dissipation in continuous medium which partly depends on the second invariant of the Cauchy tensor, a term which does not exist in discrete mechanics. Among all the differences that have been shortly highlighted and discussed, the present work aims at trying to provide a set of differences between discrete and continuum mechanics so as to discuss specific characters of turbulence. Another aim of the present work is to convince the reader that despite the fundamental different nature of the discrete mechanics formulation, classical turbulent flow characteristics are nicely recovered and modeling issues can be revisited thanks to DM approach.

The present article is structured as follows. The second section provides a synthetic description of geometrical features of discrete mechanics together with a review of mathematical formulations, modeling issues and standard numerical aspects. The third section is devoted to discussing several interesting aspects of turbulence, i.e. the turbulent pressure, 2D turbulence, inertia, viscous dissipation and the divergence of the Lamb vector, linked to the differences between DM and Navier-Stokes equations. The solid rotational motion is first considered in section 4 for illustrating and discussing the difference between DM and continuous media on a simple problem. The direct numerical simulation of the turbulent planar channel flow at turbulent Reynolds number of $R e_{\tau}=590[13,3]$ is then investigated in section 5. In particular, comparisons to DNS and LES results of the literature are discussed and fitting laws for the mean velocity profile from the wall to the center of the channel are obtained. Conclusions are finally drawn in section 6.

\section{Discrete Mechanics formulation}

\subsection{Geometric approach}

Discrete mechanics reformulates the fundamental law of dynamics from only two Galileo's intuitions, the principle of weak equivalence (WEP) and the notion of relativity. The concepts of continuous medium, one-point derivation, analysis, ... are abandoned in the favor of a geometric description where, for example, velocity and acceleration are represented by a quantity $\mathbf{V}$ or $\gamma$ assumed constant on a rectilinear oriented segment $\Gamma$ delimited by its two ends $a$ and $b$ (figure 1). The local referential $(\mathbf{m}, \mathbf{n}, \mathbf{t})$ allows you to express the different vectors, velocity, acceleration, rotation, and so on. The derivation of the discrete mechanics is developed in a preliminary work [1].

The principle of equivalence applied to the fundamental law of classical mechanics $\mathbf{F}=m \boldsymbol{\gamma}$ in the case of a force of gravitational origin makes it possible to write $m \mathbf{g}=m \boldsymbol{\gamma}$ and to suppress the mass 


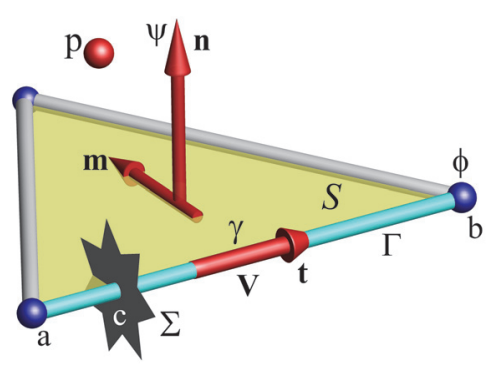

Figure 1. Elementary geometrical structure of discrete media on the local referential $(\mathbf{m}, \mathbf{n}, \mathbf{t})$ : three straight $\Gamma$ edges delimited by dots define a planar face $\mathcal{S}$. The unit normal vectors $\mathbf{n}$ to the face and the vector carried by $\Gamma$ are orthogonal, $\mathbf{t} \cdot \mathbf{n}=0$. The edge $\Gamma$ can be intercepted by a discontinuity $\Sigma$ located in $c$, between the ends $a$ and $b$ of $\Gamma . \phi$ and $\Psi$ are the scalar and vector potentials respectively. The physical medium or the particle $p$ have localized velocity and acceleration components on the edges $\Gamma$.

since this one is the same on both sides of the equality; contrary to the theory of relativity, which defines a mass at rest and another moving in the context of a change of reference, discrete mechanics defines only a local reference frame and all the interactions are of cause and effect.

Two motivations are at the origin of the suppression of mass in the equation of motion. The first relates to the gravitational attraction of light for a massless particle, the photon; thus the fundamental equation of dynamics will also be extended to objects of zero mass, for example vacuum. The second argument is related to the dimensions of all physical quantities encountered in mechanics: all those which involve the mass are at the order one; it is then possible to redefine analogous quantities per unit mass. Finally, since gravitation is only a special case of acceleration, the quantity $\mathrm{g}$ will become a generic acceleration; the fundamental equation of discrete mechanics can therefore be written:

$$
\gamma=\mathbf{g}
$$

where $\mathrm{g}$ is the sum of the forces per unit mass, $\mathrm{g}$ is an acceleration.

This law (1) is expressed by " the acceleration of a particle or a material medium with or without mass is equal to the sum of the accelerations which are applied to it ". It will always be possible to return to quantities depending on the mass, energy for example. Writing $e=m c^{2}$ or $\phi=e / m=c^{2}$ does not change physics.

The acceleration $\gamma$ of a particle, a flux of particles or a material is considered in discrete mechanics as an absolute quantity attached to the single rectilinear segment $\Gamma$; the acceleration $g$ is the sum of the external accelerations defined similarly on the segment $\Gamma$. For acceleration the principle of vector summation in the mathematical sense applies without reserve, whatever the velocity. Besides, the velocity $\mathbf{V}$ will be only a secondary quantity and the filtering of a uniform velocity superimposed on $\mathbf{V}$ will allow to completely satisfy the principle of Galilean relativity.

The decomposition of a vector into an irrotational part and another solenoid will be postulated and applied to the acceleration $\gamma$ :

$$
\gamma=-\nabla \phi+\nabla \times \psi
$$


where $\phi$ is the scalar potential and $\psi$ the vector potential of the acceleration. It is always possible to define the potentials of any quantity, velocity for example, but only acceleration, an absolute quantity, will have a physical reality. The potentials of (2) are only defined to harmonic functions and depend on the boundary conditions. As the decomposition does not bring anything, the potentials $\phi$ and $\psi$ must be expressed according to the same variable and it will be the local velocity $\mathbf{V}$ even if it will remain a amount of work; only the variables $(\gamma, \phi, \psi)$ will define the evolutions of the considered system. The potentials can be expressed as a function of $p=\rho \phi$, the pressure and $\boldsymbol{\omega}=\rho \boldsymbol{\psi}$, the shear/rotation stress but the principle of equivalence allows removing the density from the equation of motion to become an equation on acceleration.

\subsection{Discrete Mechanics motion equation}

The discrete motion equation is derived from the conservation equation of acceleration (2) by expressing the deviations of potentials $\phi$ and $\psi$ as a function of velocity $\mathrm{V}$. These "deviators" are obtained on the basis of the physical analysis of the storage-destocking processes of compression and shear energies; the first is written as the divergence of velocity and the second as a dual rotational of velocity. The physical modeling of these terms is developed in a book devoted to discrete mechanics [1].

The vectorial equation of the movement and its upgrades is written as:

$$
\left\{\begin{array}{l}
\boldsymbol{\gamma}=-\nabla\left(\phi^{o}-d t c_{l}^{2} \nabla \cdot \mathbf{V}\right)+\nabla \times\left(\boldsymbol{\psi}^{o}-d t c_{t}^{2} \nabla \times \mathbf{V}\right)+\mathbf{g} \\
\alpha_{l} \phi^{o}-d t c_{l}^{2} \nabla \cdot \mathbf{V} \longmapsto \phi^{o} \\
\alpha_{t} \boldsymbol{\psi}^{o}-d t c_{t}^{2} \nabla \times \mathbf{V} \longmapsto \boldsymbol{\psi}^{o} \\
\mathbf{V}^{o}+\gamma d t \longmapsto \mathbf{V}^{o}
\end{array}\right.
$$

The quantities $\phi^{o}$ and $\psi^{o}$ are the equilibrium potentials, the same ones that allow the equation to be satisfied exactly at the discrete instants $t$ and $t+d t ; c_{l}$ and $c_{t}$ are the longitudinal and transverse celerities, intrinsic quantities of the medium that can vary according to physical parameters. The terms $d t c_{l}^{2} \nabla \cdot \mathbf{V}$ and $d t c_{t}^{2} \nabla \times \mathbf{V}$ are respectively the deviators of the compression and shear effects. The second member is thus composed of two oscillators $\phi^{o}$ and $\boldsymbol{\psi}^{o}$ which represent energies per unit mass exchanging these with their respective deviators. The two terms in gradient and in dual rotation are orthogonal and can not exchange energy directly; if an imbalance due to an external event occurs for one of these effects, then the acceleration is changed and the energy is consequently redistributed to the other term. The acceleration $g$ represents gravity or any other source quantity and will also be written in the form of a Hodge-Helmholtz decomposition.

The physical parameters $\alpha_{l}$ and $\alpha_{t}$ are the attenuation factors of the compression and shear waves. They also depend only on the medium considered, for example a Newtonian fluid retains the shear stresses only for very weak relaxation time constants, of order of magnitude of $10^{-12} s$ and the factor $\alpha_{t}$ can be taken as zero. The updating of potentials at time $t+d t$ is thus affected by these coefficients ranging between zero and unity. The velocity and possibly the displacement $\mathbf{U}$ are upgraded in turn. In the case where the density is not constant, it is also updated using the mass conservation in the form $\rho=\rho^{o}-d t \rho^{o} \nabla \cdot \mathbf{V}$, 
this quantity is only an explicit function of the divergence of velocity.

It should be noted that $\phi^{o}$ and $\psi^{o}$ are energies per unit mass, each of the two terms reflects the behavior of the medium or particle with respect to longitudinal and transverse waves. The term $\left(\phi^{o}-d t c_{l}^{2} \nabla \cdot \mathbf{V}\right)$ represents the longitudinal wave where $\phi^{o}$ accumulates the compression energy contained in the deviator term or restores it over time according to $c_{l}$. Similarly $\left(\psi^{o}-d t c_{t}^{2} \nabla \times \mathbf{V}\right)$ is the oscillator corresponding to the velocity transverse waves $c_{t}$.

The time interval between two observations of the physical system in equilibrium is arbitrary, for example $d t=10^{20} s$ to obtain a stationary state whereas $d t=10^{-20} s$ for the study in direct simulation of $\gamma$ rays. The solution does not depend on this parameter if it is adapted to the mimicked physics, in the general case the solution is of order two in space and time. Since all the terms are implicit on the variable velocity, or linearized as the terms of inertia, the system (3) is particularly robust.

The system (3) is generic and allows to mimic the phenomena of solid mechanics, fluid mechanics, electromagnetism or optics without any modification; the properties, $\left(c_{l}, c_{t}, \alpha_{l}, \alpha_{t}\right)$ are of course specific to the media considered and can vary over several tens of orders of magnitude. In fact it is, for example, the product $d t c_{l}^{2}$ which is significant of the phenomenon considered; Thus, for a gas flow, the time to be retained is $d t \approx 1 / c_{l}^{2} \approx 10^{-5} \mathrm{~s}$, whereas for visible light, it is necessary to keep a lapse of time small $d t \approx 1 / c_{l}^{2} \approx 10^{-17} s$. The resolution of this system can be achieved with the usual variables of each of the concerned domains or in terms of potentials. It is possible to return at any time to the classical variables of mechanics, fluid and solid, or electromagnetism but the return to the variables of the represented domains is however not required, the solution of a problem depends in fact only on variables $(\mathbf{V}, \phi, \boldsymbol{\psi})$ and of course the intrinsic properties of studied environments; we note that the latter often involved in the form of products of properties conventionally used.

As the velocity the potentials $\phi$ and $\psi$ must be advected, the writing of the system (3) makes it possible to separate the Lagrangian phase of upgrades for the potentials to the Eulerian advection phase, which is of a completely different physical meaning.

\section{On a discrete approach of turbulence}

Turbulence in fluid flows is arguably one of the most complex problems that mechanics has ever attempted to understand and model. In an incompressible continuous medium with constant physical properties, the Navier-Stokes equations are able to describe any of the phenomena that can be observed in a turbulent flow. Direct simulations based on these equations cannot be faulted for their representativeness - they allow us to understand these physical phenomena and model them. However, there are differences between the Navier-Stokes equations and the discrete equations of motion, some of which are identified and discussed in the present article. In particular, there are differences in the concept of viscous dissipation on which the standard models of turbulence are based, as well as the non-validity of Stokes' hypothesis. Given the small spatial and time scales of turbulent phenomena, the compression viscosity plays an extremely important role. Moreover, the interactions between vortices at different scales favor an approach that emphasizes the curl. Despite its benefits, the direct approach of the discrete mechanics will be left to one side to instead formulate turbulence from the model of a well-understood reference case, the planar channel flow. 


\subsection{On importance of the turbulent pressure}

From Prandtl's mixing length model to much more sophisticated models with $n$ equations, the objective of any turbulence model is to find an expression for the Reynolds tensor, which is defined by decomposing the instantaneous velocity into an average value and a fluctuation. Every turbulence model is based on the Navier-Stokes equations, which are either solved directly (DNS), by separating the scales of the turbulence (LES) [17], or by statistical modeling; there are many variants of these techniques, each with its own specific advantages and disadvantages.

However, the derivation of the Navier-Stokes equations introduces various obstacles and generate artifacts that are sometimes extremely significant. One example of a problem is the fact that the compression viscosity is defined inappropriately, usually with Stokes' hypothesis, $3 \lambda+2 \mu=0$. The Clausius-Duhem inequality cannot be used to determine the value of the compression viscosity. These flaws and defects are partially resolved by introducing a strong coupling between the conservation law and the state equations, at the cost of requiring additional assumptions. In some specific cases, together with these supplementary assumptions, the Navier-Stokes equations succeed in establishing a model that is representative of reality - for example in incompressible problems with constant properties. Nonetheless, in the general case, some caution is required.

Approaches to modeling the turbulence other than by direct simulation are usually based entirely on the viscous effects. The turbulent pressure term introduced into the gradient doesn't make any more sense than Stokes' law. Furthermore, no equilibrium is guaranteed between the pressure and viscous effects. The simplest models are forced to introduce terms that do not appear in the equations of motion to describe the production and dissipation of turbulence. But the dissipation does not appear in the equations of motion, and the viscous diffusion has nothing to do with the dissipation.

In the next part of this section, we shall establish a turbulence model based on discrete mechanics. The turbulent equations of motion are derived from Newton's second law [14] and the hypothesis that there is equivalence between the gravitational mass and the inertial mass. Accordingly, the equations of motion are no longer phrased in terms of the conservation of momentum, but rather the conservation of the acceleration. Formally, the equations of motion are expressed as a Hodge-Helmholtz decomposition, namely the sum of the gradient of a scalar function and the curl of a vector function. For incompressible problems with constant properties, it can be shown that these discrete equations reduce to the NavierStokes equations and have strictly the same solutions; however, the same is not true in the general case. The discrete equations of motion only have two physical parameters - the compressibility coefficient and the viscosity. Both are perfectly measurable in both fluids and solids (where $\mu$ is the shear modulus).

Can these equations of motion reproduce the mean stationary solution of a turbulent flow without re-

quiring any modifications to their structure or additional terms? They can certainly produce the unsteady turbulent solution by direct simulation (DNS) in the same way as the Navier-Stokes equations. Below, we will see that the discrete equations can also find the average solution. The only parameter that we need to modify is the apparent viscosity of the turbulent flow. By analyzing the DNS solutions recorded in various databases, we can determine the value of the discrete turbulent viscosity using the differential geometry operators derived from the equations of motion. 


\subsection{Dynamics of the vorticity in two spatial dimensions}

Vortex interactions play an essential role in turbulent flows. A turbulent flow is effectively a platform for intricately entwined and highly unsteady vortex structures whose curl vectors are arbitrarily oriented in every direction of space. Furthermore, when the Reynolds number characterizing the ratio of the molecular convection and diffusion forces is high, there can be a wide spectrum of vortices of different sizes and frequencies. The largest vortices occupy the lower-frequency regions of the energy spectrum and are primarily influenced by the boundary conditions of the problem. The size of these vortices is typically of the order of magnitude of the domain containing the fluid. The smaller vortices occupy the higher-frequency regions of the spectrum. Their size is determined by the viscous forces - these smaller vortices are responsible for dissipating the turbulent energy. The relative size difference between the larger and smaller structures is proportional to the overall Reynolds number of the flow. At higher Reynolds numbers, the energy spectrum becomes broader and we encounter finer and finer structures. This is the reason why turbulent flows can become challenging in various way as the Reynolds number increases.

All of these classical ideas about turbulence are analyzed in various reference works, such as the book by Pope [15]. We will only focus on one specific aspect here, namely that some of the differences between 2D and 3D turbulence in a continuum disappear in a discrete medium.

The vorticity equation $\Omega=\nabla \times \mathbf{V}$ may be established by applying the curl operator to the equations of motion - in other words, the Navier-Stokes equations in continuum mechanics:

$$
\left\{\begin{array}{l}
\rho\left(\frac{\partial \mathbf{V}}{\partial t}+\mathbf{V} \cdot \nabla \mathbf{V}\right)=-\nabla p+\nabla \cdot\left(\mu\left(\nabla \mathbf{V}+\nabla^{t} \mathbf{V}\right)\right) \\
\nabla \cdot \mathbf{V}=0
\end{array}\right.
$$

One effect of applying an operator such as the divergence (or the curl) to these vector equations (1) - and indeed any other equation - is that any curl (or gradient) terms are eliminated. Even though this operation is algebraically justified, it changes the nature of the equilibrium between the physical phenomena represented by these terms. Furthermore, this operation makes it desirable to assume that the physical parameters of equation (1), namely the density and the viscosity, are constant, otherwise we would acquire various additional terms arising from the derivatives of these quantities. From the vector relation $\nabla \times(\mathbf{V} \cdot \nabla \mathbf{V})=\mathbf{V} \cdot \nabla \Omega-\Omega \cdot \nabla \mathbf{V}+\Omega \nabla \cdot V$, we can deduce the classical vorticity equation:

$$
\frac{\partial \boldsymbol{\Omega}}{\partial t}+\mathbf{V} \cdot \nabla \boldsymbol{\Omega}-\boldsymbol{\Omega} \cdot \nabla \mathbf{V}+\boldsymbol{\Omega} \nabla \cdot \mathbf{V}=\nu \nabla^{2} \boldsymbol{\Omega}
$$

In two spatial dimensions, writing the velocity as $\mathbf{V}=u(x, y) \mathbf{e}_{x}+v(x, y) \mathbf{e}_{y}$, the term $\boldsymbol{\Omega} \cdot \nabla \mathbf{V}$ vanishes, since the curl is orthogonal to the gradient vector of the velocity in the $(x, y)$-planar surface. If we also write $\omega=\Omega \cdot \mathbf{e}_{z}$, then the vorticity equation of an incompressible flow becomes:

$$
\frac{\partial \omega}{\partial t}+\mathbf{V} \cdot \nabla \omega=\nu \nabla^{2} \omega
$$

The term describing the vortex stretching by the velocity gradient has been eliminated, which makes Equation (3) an advection-diffusion equation for the scalar $\omega$. We can add another equation to the sole- 
noidal vector potential $\Psi$ defined by $\mathbf{V}=\nabla \times \Psi$, or more precisely to the stream function $\psi$ defined by $\boldsymbol{\Psi}=(0,0, \psi)$. The quantities $\omega$ and $\psi$ are pseudo-scalars. For a kinematic viscosity $\nu=0$, the equation becomes an advection equation that can be rewritten as $d \omega / d t=0$. Thus, the vorticity is conserved throughout the motion of an element of matter. Various other quantities are also conserved in this case, including the kinetic energy and the enstrophy:

$$
\left\{\begin{array}{l}
E_{c}=\frac{1}{2} \iiint_{\Omega}\|\mathbf{V}\|^{2} d v \\
E_{n}=\frac{1}{2} \iiint_{\Omega}\|\boldsymbol{\Omega}\|^{2} d v
\end{array}\right.
$$

The fact that the enstrophy is conserved in two dimensions means that it is impossible for energy to dissipate. This is the key difference between 2D and 3D turbulence. The whole of continuum mechanics revolves around the axiom that effects must unfold in the same planar surface as their actions; for example, a velocity gradient in the $(x, y)$-planar surface can only be established from a shearing action in this same planar surface.

In discrete mechanics, there is no such distinction between two and three dimensions. The gradient, divergence, primal curl, and dual curl operators are three-dimensional by their very nature. These operators respectively allow us to define vectors by reference to the oriented bipoint $\Gamma$ shown in Figure 1, recover a scalar assigned to a point, calculate the circulation of a vector defined along the normal of an oriented surface, and recover an oriented bipoint. To study a formulation of the vorticity, let us return to the discrete equations of motion:

$$
\frac{\partial \mathbf{V}}{\partial t}-\nabla_{d} \times\left(\frac{\|\mathbf{V}\|^{2}}{2} \mathbf{n}\right)+\nabla\left(\frac{\|\mathbf{V}\|^{2}}{2}\right)=-\nabla \phi+\nabla_{d} \times\left(\nu \nabla_{p} \times \mathbf{V}\right)
$$

where the indices $p$ and $d$ identify the primal and dual curl operators; the first is defined along the normal $\mathbf{n}$ of each face and calculates the circulation of the velocity vector along the primal contour $\Gamma$, and the second projects the result of the circulation along the dual contour onto the edge $\Gamma$.

Suppose now that we apply the primal curl operator to Equation (5). Writing $\Omega=\nabla_{p} \times \mathbf{V}$ and $\phi_{i}=\|\mathbf{V}\|^{2} / 2$ for the inertial potential, we find:

$$
\frac{\partial \boldsymbol{\Omega}}{\partial t}-\nabla_{p} \times \nabla_{d} \times\left(\phi_{i} \mathbf{n}\right)=\nabla_{p} \times \nabla_{d} \times(\nu \boldsymbol{\Omega}) .
$$

The second term could be rewritten using the relation $\nabla_{d} \times\left(\phi_{i} \mathbf{n}\right)=\phi_{i} \nabla_{d} \times \mathbf{n}+\nabla \phi_{i} \times \mathbf{n}$, but the conclusions remain the same. Applying the primal curl to this term does not eliminate either of its components; if there are no viscous terms $(\nu=0)$, we now obtain:

$$
\frac{\partial \boldsymbol{\Omega}}{\partial t}-\nabla_{p} \times \nabla_{d} \times\left(\phi_{i} \mathbf{n}\right)=0
$$


The nature of these equations is the same in both two and three dimensions. Both terms are topologically consistent, since the vorticity $\Omega$ is defined along the normal $\mathbf{n}$ of each face, and so is the contribution $\nabla_{p} \times \nabla_{d} \times\left(\phi_{i} \mathbf{n}\right)$. The two quantities are both pseudovectors. Thus, in discrete mechanics, it doesn't make sense to relate the dimension of space to the turbulence. If there are any differences, we can perform homogeneous isotropic turbulence simulations to characterize them. In light of this, Equation (6) is not useful and the original formulation (5) is the only suitable approach for direct simulations. The physical viscous dissipation characterized by the term $\nu\left(\nabla_{p} \times \mathbf{V}\right)^{2}$ is a different topic that is discussed elsewhere. If the viscosity is zero, there cannot be viscous dissipation, whether in $2 \mathrm{D}$ or in 3D.

\subsection{Formulation of turbulent dissipation}

There are other formal differences between a continuum and a discrete medium, such as the dissipation of waves, either by attenuation or by viscous stresses. In continuum mechanics, the dissipation function is given by:

$$
\phi_{d}=(\lambda \nabla \cdot \mathbf{V} \mathbf{I}+2 \mu \mathbf{D}): \nabla \mathbf{V}
$$

The double contracted product of the identity tensor $\mathbf{I}$ with $\nabla \mathbf{V}$ gives the divergence of the velocity:

$$
\phi_{d}=\lambda(\nabla \cdot \mathbf{V})^{2}+\mu\left(\nabla \mathbf{V}+\nabla^{t} \mathbf{V}\right): \nabla \mathbf{V}
$$

which is equal to:

$$
\phi_{d}=\lambda(\nabla \cdot \mathbf{V})^{2}+\mu|\nabla \mathbf{V}|^{2}+\mu \nabla^{t} \mathbf{V}: \nabla \mathbf{V}
$$

Noting that $\|\nabla \mathbf{V}\|^{2}=(\nabla \cdot \mathbf{V})^{2}+(\nabla \times \mathbf{V})^{2}$, we have:

$$
\phi_{d}=(\lambda+\mu)(\nabla \cdot \mathbf{V})^{2}+\mu(\nabla \times \mathbf{V})^{2}+\mu \nabla^{t} \mathbf{V}: \nabla \mathbf{V} .
$$

Since $\nabla^{t} \mathbf{V}: \nabla \mathbf{V}=(\nabla \cdot \mathbf{V})^{2}-2 I_{2}$, this gives us an expression for the local dissipation function:

$$
\phi_{d}=(\lambda+2 \mu)(\nabla \cdot \mathbf{V})^{2}+\mu(\nabla \times \mathbf{V})^{2}-2 \mu I_{2}
$$

where $I_{2}$ is the second invariant of the tensor $\nabla \mathbf{V}$ :

$$
I_{2}=\frac{1}{2}\left[(\operatorname{tr}(\nabla \mathbf{V}))^{2}-\nabla \mathbf{V}: \nabla \mathbf{V}\right]=\frac{1}{2}\left[(\operatorname{tr}(\nabla \mathbf{V}))^{2}-\operatorname{tr}\left((\nabla \mathbf{V})^{2}\right)\right]
$$

Continuum mechanics shows that, provided that Stokes' hypothesis $3 \lambda+2 \mu \geq 0$ is satisfied, the dissipation function is always a positive quantity; in thermodynamics, the Clausius-Duhem inequality leads to the same result. The inverse of $(\lambda+2 \mu)$ is in fact just the compressibility of the medium $\chi_{T}$. The first two terms of Equation (12) are positive, whereas $I_{2}$ can be either positive or negative locally. The dissipation function itself, however, is always positive or zero. 
On a separate point of view, the term $\mu \nabla \cdot \mathbf{V}$ involves two quantities, the shear viscosity and an operator associated with the variation of volume. These two concepts are entirely distinct. The connection between them is explained by the historical path taken by mechanics: when an extension is applied to a sample of a ductile material in a certain direction, the sample experiences a tangential stress and a retraction in the other directions. This effect was historically interpreted as shearing, motivating a threedimensional perspective and the use of tensors - but this is in fact a misunderstanding of the nature of the phenomenon. Any shearing in this example is exclusively governed by the lateral boundary conditions of the sample and the conservation of mass.

If we remain strictly within the scope of continuum mechanics, certain quantities can be calculated in an orthogonal Cartesian frame. Consider the quantities $(\nabla \times \mathbf{V})^{2}$ and $(\nabla \cdot \mathbf{V})^{2}$ in the $(x, y)$-planar surface, where $\mathbf{V}=u(x, y) \mathbf{e}_{x}+v(x, y) \mathbf{e}_{y}$ :

$$
\left\{\begin{array}{l}
(\nabla \times \mathbf{V})^{2}=\left(\frac{\partial v}{\partial x}\right)^{2}+\left(\frac{\partial u}{\partial y}\right)^{2}-2\left(\frac{\partial v}{\partial x} \frac{\partial u}{\partial y}\right) \geq 0 \\
(\nabla \cdot \mathbf{V})^{2}=\left(\frac{\partial u}{\partial x}\right)^{2}+\left(\frac{\partial v}{\partial y}\right)^{2}+2\left(\frac{\partial u}{\partial x} \frac{\partial v}{\partial y}\right) \geq 0
\end{array}\right.
$$

The first expression leads to the classical inequality $a^{2}+b^{2} \geq 2 a b$. Summing both operators together gives:

$$
\begin{array}{r}
(\nabla \times \mathbf{V})^{2}+(\nabla \cdot \mathbf{V})^{2}=\left(\frac{\partial v}{\partial x}\right)^{2}+\left(\frac{\partial u}{\partial y}\right)^{2}+\left(\frac{\partial u}{\partial x}\right)^{2}+\left(\frac{\partial v}{\partial y}\right)^{2} \\
-2\left(\frac{\partial v}{\partial x} \frac{\partial u}{\partial y}-\frac{\partial u}{\partial x} \frac{\partial v}{\partial y}\right)
\end{array}
$$

This last term of right-hand side is in fact the component in the $(x, y)$-planar surface of $I_{2}$, the second invariant of the second-order tensor $\nabla \mathbf{V}$. In general, for a three-dimensional Cartesian space $I_{2}$ has the expression:

$$
I_{2}=\left(\frac{\partial u}{\partial z} \frac{\partial w}{\partial x}+\frac{\partial v}{\partial z} \frac{\partial w}{\partial y}+\frac{\partial u}{\partial y} \frac{\partial v}{\partial x}-\frac{\partial u}{\partial x} \frac{\partial v}{\partial y}-\frac{\partial v}{\partial y} \frac{\partial w}{\partial z}-\frac{\partial w}{\partial z} \frac{\partial u}{\partial x}\right)
$$

In summary, differentiating introduces a perturbation when $I_{2}$ is locally positive, but the full expression (16) is always positive.

In discrete mechanics, the developed expressions of the operators are not used and $(\nabla \cdot \mathbf{V})^{2}$ is a positive scalar; the quantity $(\nabla \times \mathbf{V})^{2}$ on the other hand is a scalar product with norm $\|\nabla \times \mathbf{V}\|^{2}$. Hence, the quantity $(\nabla \times \mathbf{V})^{2}+(\nabla \cdot \mathbf{V})^{2}$ is intrinsically positive. These operators should be viewed as elementary quantities that cannot be developed in any specific frame of reference and which have a clear geometric meaning in their own right. In discrete mechanics, the dissipation function is given by:

$$
\phi_{d}=\frac{d t}{\chi_{T}}(\nabla \cdot \mathbf{V})^{2}+\mu(\nabla \times \mathbf{V})^{2}
$$


where $\chi_{T}$ and $\mu$ are perfectly measurable positive properties in every medium; there are no conditions on the equations of discrete mechanics. In a fluid, $\mu$ is the shear viscosity; in a solid, $\mu$ becomes the shear modulus. The physical parameter describing the compression is $d t / \chi_{T}$ in both solids and fluids where this stress is accumulated. In discrete mechanics, every term in the equations of motion is an acceleration. The dissipation function per unit mass can be written in the following generic form:

$$
\phi_{d m}=d t c_{l}^{2}(\nabla \cdot \mathbf{V})^{2}+d t c_{t}^{2}(\nabla \times \mathbf{V})^{2} .
$$

The longitudinal celerity $c_{l}$ and the transverse celerity $c_{t}$ are intrinsic properties of the medium that may depend on other quantities such as the temperature. The first term in the right-hand side of Equation (18) is the part of the dissipation of waves that is attributable to attenuation, and the second term is the dissipation induced by shearing or differential rotation. Continuum mechanics and discrete mechanics adopt two different perspectives that lead to the same results; the formal differences that can be observed between them, such as those cited here relating to the dissipation, arise from the discrete-mechanical axiom that the compression and rotation effects are fully separable.

\subsection{On the divergence of acceleration}

In the Navier-Stokes equations, the inertial term can be written according to Lamb vector [9], $\mathcal{L}=$ $\nabla \times \mathbf{V} \times \mathbf{V}$. If we are particularly interested in the divergence of this vector as authors like Marmanis, [12], Hamman \& al. [4], Lindgren [10] or Kozachock [8] have been, it can be demonstrated that $\nabla \cdot \mathcal{L}=$ $\mathbf{V} \cdot \nabla \times \nabla \times \mathbf{V}-\|\nabla \times \mathbf{V}\|^{2}$. In this expression, the first term is called flexion whereas the second one is the local enstrophy. A rigid rotation movement thus generates local enstrophy as the rotational motion is at zero divergence but non-zero rotational $\nabla \times \mathbf{V}=2 \omega$. In the present section, the purpose is to examine the consequences of the expression of the divergence of the Lamb vector on that of the acceleration $\gamma$.

If the solutions of the equations of the discrete mechanics are the same as those of the Navier-Stokes equations with constant properties, it turns out that other differences appear when the term of dissipation is extracted from these equations or if the divergence operator is applied to the acceleration. Consider here the divergence operator applied to the motion equation $\gamma=-\nabla \phi+\nabla \times \boldsymbol{\psi}$, with leads to $\nabla \cdot \gamma=$ $-\nabla^{2} \phi$.

The difference between continuum mechanics and discrete mechanics relates to the fact that $\nabla \cdot \gamma=$ $\nabla \cdot(d \mathbf{V} / d t)$. In discrete mechanics, the particular derivative writes

$$
\frac{d \mathbf{V}}{d t}=\frac{\partial \mathbf{V}}{\partial t}+\frac{1}{2} \nabla\left(\|\mathbf{V}\|^{2}\right)-\frac{1}{2} \nabla \times\left(\|\mathbf{V}\|^{2} \mathbf{n}\right)
$$

and the divergence of it reads

$$
\nabla \cdot\left(\frac{d \mathbf{V}}{d t}\right)=\nabla \cdot\left(\frac{\partial \mathbf{V}}{\partial t}\right)+\frac{1}{2} \nabla^{2}\left(\|\mathbf{V}\|^{2}\right)
$$

In the framework of continuum mechanics, the inertial term coming from the particular derivative writes $\mathbf{V} \cdot \nabla \mathbf{V}$ or $-\mathbf{V} \times \nabla \times \mathbf{V}+\nabla\left(\|\mathbf{V}\|^{2} / 2\right)$. By considering one of these two forms of the inertial terms, the divergence operator applied to them provides the same expression: 


$$
\nabla \cdot\left(\frac{d \mathbf{V}}{d t}\right)=\nabla \cdot\left(\frac{\partial \mathbf{V}}{\partial t}\right)+\mathbf{V} \cdot \nabla(\nabla \cdot \mathbf{V})+(\nabla \cdot \mathbf{V})^{2}-2 I_{2}
$$

where the term $I_{2}$ is the second invariant of the velocity gradient presented in the previous section (16).

Is it possible to define a pseudo-vector $\boldsymbol{I}$ where components are associated to each surface defined $t_{i}$, $t_{j}$, the unit vectors on each axis for the three directions of the space in Cartesian coordinates $I_{2}$ is then defined as the scalar product $\boldsymbol{I}$ by the normal $\mathbf{n}=t_{i} \times t_{j}$ to each of the planar surfaces of the system.

Although $I_{2}$ is a scalar, each of the components of vector $\boldsymbol{I}$ has to be zero so that $\nabla \cdot \gamma$ be zero too if the flow motion is incompressible $\nabla \cdot \mathbf{V}=0$. The constraints $\boldsymbol{I}=0$ are presented as compatibility conditions to be satisfied by component. They are similar to those used for defining displacements from constraints in solid continuum mechanics.

In discrete mechanics the notion of global referential does not exist, it is replaced by a local reference frame of a space with $n$ dimensions. If $u$ and $v$ are the components of the velocity in a surface then the term $-2 I_{2}$ can be represented by $\nabla u \wedge \nabla v$ where the gradient operator is applied in the plane $(x, y)$ and where $\wedge$ is the external product carried by the normal to this surface.

\section{Rigid rotational motion}

Rigid rotational motion leads to consistency problems in $\mathcal{R}^{3}$. Nonetheless, it is an essential problem, and any viable model must be capable of fully reproducing its behavior. Continuum mechanics falls short in this regard; the divergence of the Lamb vector $\nabla \cdot \mathcal{L}$ generates local enstrophy, since $\nabla \cdot(\nabla \times \mathbf{V} \times \mathbf{V})=\mathbf{V} \cdot \nabla \times \nabla \times \mathbf{V}-(\nabla \times \mathbf{V})^{2}$, where the first term is known as the flexion and the second term is the enstrophy [4]. The enstrophy is non-zero for rigid rotations, since any rotational motion has zero divergence and non-zero curl $\nabla \times \mathbf{V}=2 \Omega \mathbf{n}$, where $\Omega$ is the rotational velocity. The same obstacle is encountered in the expression of the dissipation function $\phi_{d}$, where $I_{2}$, the second invariant of the second-order tensor $\nabla \mathbf{V}$, appears as an additional, artifactual term in the dissipation [1]. For rigid motion with local velocity $\mathbf{V}_{\theta}=\Omega r \mathbf{e}_{\theta}$, dissipation cannot occur.

In this case, it is straightforward to compute the components of the Lamb vector, the gradient term, and the curl of $\phi_{i} \mathbf{n}$ in polar coordinates:

$$
\left\{\begin{array}{l}
-\mathbf{V} \times \nabla \times \mathbf{V}=-2 \Omega^{2} r \mathbf{e}_{r} \\
\nabla \phi_{i}=\Omega^{2} r \mathbf{e}_{r} \\
-\nabla \times \boldsymbol{\psi}_{i}=\Omega^{2} r \mathbf{e}_{\theta}
\end{array}\right.
$$

where $\phi_{i}=\|\mathbf{V}\|^{2} / 2$ and $\boldsymbol{\psi}_{i}=\phi_{i} \mathbf{n}$.

In continuum mechanics, the mechanical equilibrium is presented as a single equality on the radial component, $\mathbf{e}_{r}:-\mathbf{V} \times \nabla \times \mathbf{V}+\nabla\left(\|\mathbf{V}\|^{2} / 2\right)=-\nabla \phi$, allowing us to find the value of the potential $\phi$ (or the pressure $p$ ), $\phi^{o}=\Omega^{2} r^{2} / 2$ by summing the first two vectors. Note that the Lamb vector is a pure gradient here. The vector $\nabla \times\left(\phi_{i} \mathbf{n}\right)$ is inconsistent; summing the second and final vector does not give 
the desired result. The term $\nabla \times\left(\phi_{i} \mathbf{n}\right)$ acts on the second component $\mathbf{e}_{\theta}$. In fact, it is impossible to express the Lamb vector as a curl in classical mechanics.

In discrete mechanics, the Lamb vector does not exist. The equations of motion are given by:

$\frac{\partial \mathbf{V}}{\partial t}+\nabla \phi_{i}-\nabla \times \boldsymbol{\psi}_{i}=-\nabla\left(\phi^{o}-d t c_{l}^{2} \nabla \cdot \mathbf{V}\right)+\nabla \times\left(\boldsymbol{\psi}^{o}-d t c_{t}^{2} \nabla \times \mathbf{V}\right)$

where the gradient and the curl of $\phi_{i}$ represent all inertial effects. The equations of motion of the rigid rotational motion at mechanical equilibrium are given by:

$$
-\nabla\left(\phi^{o}+\frac{\Omega^{2} r^{2}}{2}\right)+\nabla \times\left(\boldsymbol{\psi}^{o}+\frac{\Omega^{2} r^{2}}{2} \mathbf{n}\right)=0
$$

The vector $\boldsymbol{\psi}^{o}$ aligns with $\mathbf{n}=\mathbf{e}_{z}$, as expected; the gradient of the scalar potential projects this quantity onto $\mathbf{e}_{r}$, whereas the dual curl of the vector potential projects it onto $\mathbf{e}_{\theta}$. Since the two fields in Equation (3) are orthogonal, the mechanical equilibrium can be presented as the following two separate equations:

$$
\left\{\begin{array}{l}
-\nabla\left(\phi^{o}+\frac{\Omega^{2} r^{2}}{2}\right)=0 \\
\nabla \times\left(\boldsymbol{\psi}^{o}+\frac{\Omega^{2} r^{2}}{2} \mathbf{n}\right)=0
\end{array}\right.
$$

This enables us to find the solution of the problem, $\left(\phi^{o}=-\Omega^{2} r^{2} / 2, \boldsymbol{\psi}^{o}=-\Omega^{2} r^{2} / 2 \mathbf{n}\right)$. Here, the vector $\psi^{o}$ does not derive from an accumulation process, it simply represents an instantaneous equilibrium potential. Similarly, the scalar potential is not accumulated, since the motion is assumed to be steady and incompressible.

Does the discrete-mechanical inertial term $\nabla \phi_{i}-\nabla \times\left(\phi_{i} \mathbf{n}\right)$ enable us to solve the rigid motion in terms of velocity and pressure? The concept of mechanical equilibrium is more subtle to interpret in discrete mechanics, since we cannot refer to specific frames or components. Direct simulation is performed instead. Consider the case of a disk initially at rest, $(\mathbf{V}=0)$, experiencing a rotation induced by its outer surface, which is held at a rotational velocity of $\Omega=1$. The initial potential $\phi^{o}$ (or the pressure) is also assumed to be zero. Figure 2 shows the streamlines for an unstructured triangular mesh whose pressure is equal to the inertial potential $\phi_{i}=\|\mathbf{V}\|^{2} / 2$ times a density. Up to numerical errors, the solution reproduces the velocity field $\mathbf{V}_{\theta}=\Omega r \mathbf{e}_{\theta}$ and the potential $\phi^{o}=-\Omega^{2} r^{2} / 2$ plus some additive constant. The mechanical equilibrium is established by balancing the centrifugal and centripetal accelerations.

We therefore recover the theoretical solution with an inertial term of the form $\nabla \phi_{i}-\nabla \times\left(\phi_{i} \mathbf{n}\right)$. The Hodge-Helmholtz decomposition decouples the solenoidal and irrotational components of the inertial effects, in the same way as the other terms of the equations of motion. The restrictiveness of continuum mechanics with its underlying concept of a continuum is apparent: continuum mechanics requires us to establish the equilibrium at a point and thereby inhibits any operations that could support an implicit coupling between the variables. 


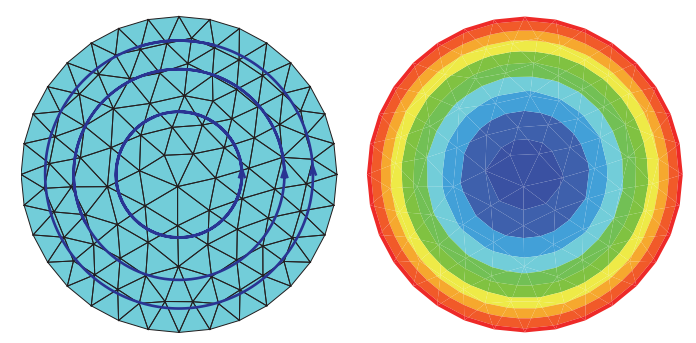

Figure 2. Rigid rotational motion: left, the unstructured mesh and the streamlines; right, the inertial potential field $\phi_{i}=\Omega^{2} \mathrm{r}^{2} / 2$

\section{Turbulent flow in channel}

\subsection{Analysis of a turbulent flow in a planar channel}

The majority of turbulence models are first validated against experimental results and then by direct simulations at moderate Reynolds numbers. More recently, databases have been compiled, allowing us to explore such flows in finer detail and develop more representative models, including problems where the production of turbulence arises from other mechanisms than just shearing.

We shall consider two databases in order to formulate the discrete model of a turbulent flow: the contributions of 7 authors to a comparison by Denaro [3] with a turbulent Reynolds number of $R e_{\tau}=$ 590, and the earlier simulations by Moser et al. [13] for the same problem.

The comparison by Denaro mainly focuses on the results of Large-Eddy Simulations (LES), however the model-free results are also presented which are obtained for meshes with points in the viscous boundary sublayer as subgrid-scale direct simulations. This will be sufficient for an initial analysis of the discrete model. The details of the configuration are stated in the synthesis [3].

Figure 3 shows the average velocity profiles in wall coordinates $u^{+}=f\left(y^{+}\right)$. We can identify zones classically described in the literature a priori: the viscous sublayer for $y^{+}<5$, the buffer zone, and the so-called logarithmic zone for $y^{+}>30$.

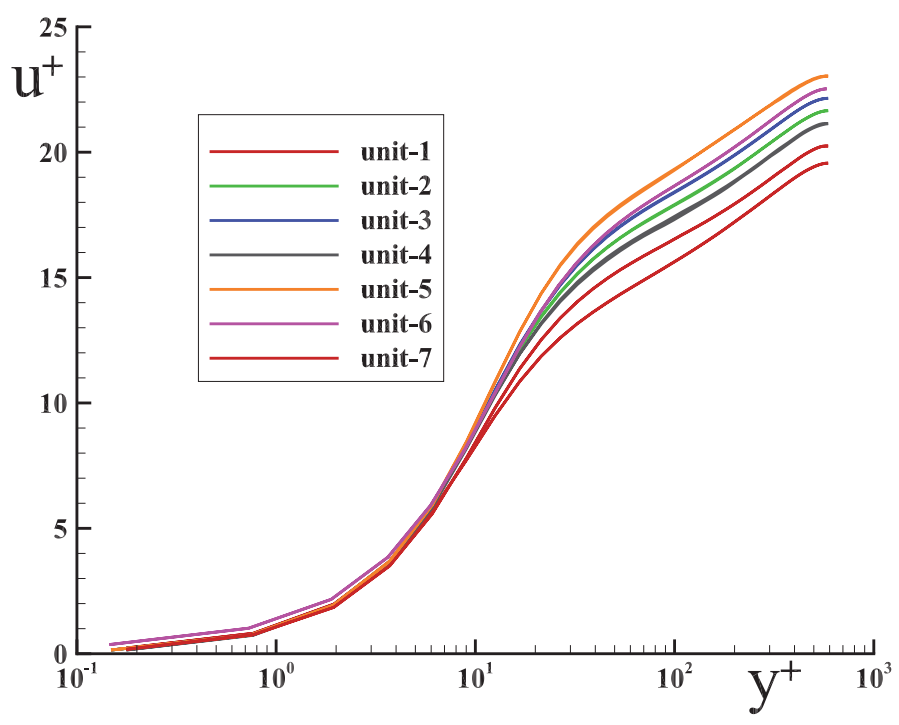

Figure 3. Turbulent channel with $\operatorname{Re}_{\tau}=590$; average velocity profiles in reduced coordinates $\mathrm{u}^{+}=$ $f\left(\mathrm{y}^{+}\right)$, deduced from the various contributions to [3] 
Starting from these average velocity profiles, we can characterize other zones in terms of the operators of discrete mechanics, including the dual curl of the primal curl $\mathbf{r}=-\nabla \times \nabla \times \mathbf{V}$. This vector points along the edge $\Gamma$ with unit vector $\mathbf{t}$. The vector $\mathbf{r}$ is therefore collinear with the component $\mathbf{V}=\mathbf{W} \cdot \mathbf{t}$, where $\mathbf{W}$ is the velocity in a Galilean frame of reference. In practice, $\mathbf{V}$ can be viewed either as a vector or as a scalar defined on the edge $\Gamma$. This concept generalizes the ideas of staggered mesh introduced by Harlow and Welch [5] for Cartesian meshes to arbitrary generalized polyhedral topologies.

Figure 4 plots the variations of $\mathbf{r}=-\nabla \times \nabla \times \mathbf{V}$ as a function of the wall coordinates $y^{+}$. We can distinguish four zones separated by the values $y^{+}=\delta_{v}, y^{+}=\delta_{l}, y^{+}=\delta_{n}$ as follows:

- $y^{+}<\delta_{v}$, a layer where only the viscosity effects are present. The value of $\mathbf{r}=-\nabla \times \nabla \times \mathbf{V}$ is of order one. It is difficult to truly characterize the behavior of this function from this example since the mesh is relatively coarse. But in general, the law of the velocity profile is known to be almost linear, $u^{+} \approx y^{+}$.

- $\delta_{v}<y^{+}<\delta_{l}$, a zone that can be calculated very precisely by simulations, where the viscosity effects remain dominant but the turbulence is no longer negligible. This zone is characterized by a minimum of the function $\mathbf{r} \cdot \mathbf{t}$ at $y^{+}=\delta_{r}=9$. While bearing in mind the relative inaccuracy in the definition of the zones $\delta_{v}, \delta_{l}$, and $\delta_{n}$, we can expect this value of $\delta_{r}=9$ to hold more generally. This will need to be confirmed by analyzing other turbulent flows with different turbulent Reynolds numbers $R e_{\tau}$.

- $\delta_{l}<y^{+}<\delta_{n}$, a zone where the turbulence is not yet homogeneous, with some remaining viscosity effects, and where $\delta_{l}=30$ is approximately equal to the inverse of the curvature of $\mathbf{r}$. On either side of this boundary, the curvature of $\mathbf{r}$ appears to be constant.

- $y^{+}>\delta_{n}$ a region sometimes called the kernel where the turbulence is fully developed. Above $\delta_{n}=200$, the value of $\mathbf{r} \cdot \mathbf{t}$ is constant. Since the curvature of the velocity profile is small in this region, direct simulations are less accurate and depend on the degree of convergence of the turbulent statistics.

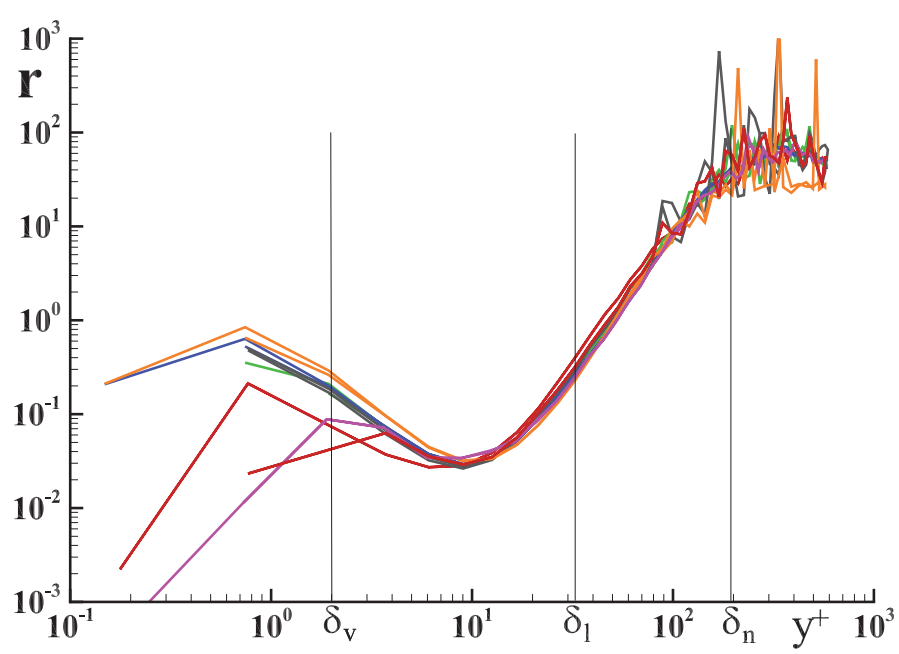

Figure 4. Turbulent channel with $\operatorname{Re}_{\tau}=590$ [3], $\boldsymbol{r}=\mathrm{f}\left(\mathrm{y}^{+}\right)=-\nabla \times \nabla \times \boldsymbol{V}$ in logarithmic coordinates

The choice of values of $\delta_{v}, \delta_{l}$, and $\delta_{n}$ will of course affect the solution, but contributions based on very different numerical methodologies (research codes, commercial software, etc.) nonetheless find 
very similar solutions. We shall choose the following boundaries between the regions described above: $\delta_{v}=2, \delta_{l}=35$, and $\delta_{n}=200$. These values allow us to reproduce the average velocity profile of the contributor unit -2 very accurately. However, the variations of these boundaries are not as accurate at representing some of the other average profiles. At this stage, it would be difficult to fix the boundaries more precisely without having better convergence toward a unique solution. Once fixed, they can be viewed as universal constants.

To gain a better understanding of the behavior of the solution in the turbulent kernel, we can plot the function $1 / \mathbf{r}=1 / \nabla \times \nabla \times \mathbf{V}$ as a function of $y^{+}$in linear coordinates. Figure 5 shows that this function is practically constant in the center of the channel. Of course, this is the region where the mesh is coarsest and the numerical errors are largest. It is also more difficult to ensure the convergence of the turbulent statistics in this region.

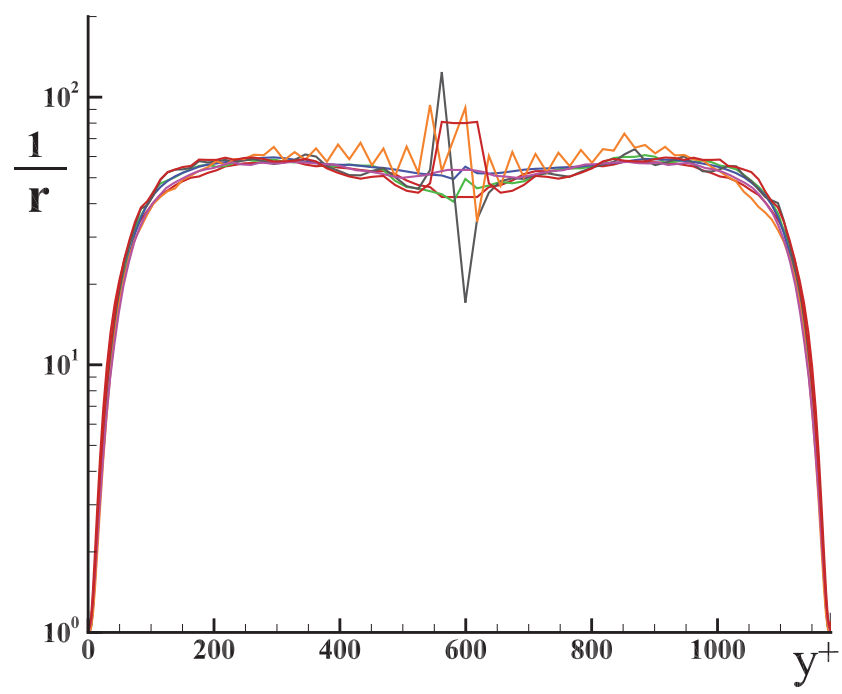

Figure 5. Turbulent channel with $\operatorname{Re}_{\tau}=590$. The function $1 / \boldsymbol{r}=\mathrm{f}\left(\mathrm{y}^{+}\right)=1 / \nabla \times \nabla \times \boldsymbol{V}$ is plotted across the width of the channel, reproduced from [3].

To analyze the behavior of the mean flow more finely, let us compare the velocity profile of the contribution unit -2 against a parabolic profile. A regression of the profile $u^{+}=f(y)$ gives us the curvature $\kappa$, which is constant. Figure 6 shows a comparison with the parabolic profile. The graph of function $u^{+}=f(y)$ on half of the channel shows that this function is strictly of order two.

The same procedure can be extended to each of the other contributions - only the curvature itself depends on the individual results. Table 5.1 lists the values of the curvature $\kappa$ for each contribution.

The table also shows the curvature found by the direct simulation by Moser et al. [13]. It is noticeably higher than the values of the model-free simulations from [3]. At this stage, the most important conclusion is that the turbulent kernel can indeed be associated with an average velocity profile with constant curvature $\kappa$.

To determine more accurate universal values for the boundaries $\delta_{v}, \delta_{l}$, and $\delta_{n}$, we need an extremely accurate set of databases. Although the mean velocity profile itself seems very smooth, the values of the first and second-order operators vary strongly, even in the direct simulations. The databases that are currently available are already sufficient to begin interpreting and analyzing the average profiles to establish a discrete-mechanical model of turbulence. 

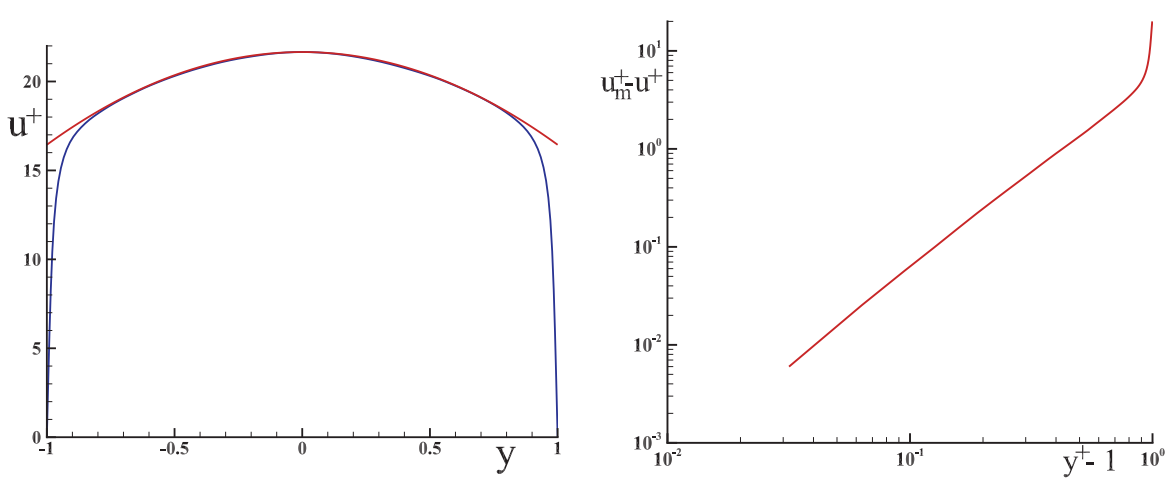

Figure 6. Turbulent channel with $\operatorname{Re}_{\tau}=590$, results of unit-2 [3]. The average velocity profile $\mathrm{u}^{+}=\mathrm{f}(\mathrm{y})$ is shown on the left, together with a parabolic profile for comparison. On the right, the same profile is plotted in logarithmic coordinates in the left half of the channel.

\begin{tabular}{|c|c|}
\hline Contribution & Curvature $\kappa$ \\
\hline \hline unit-1 & -5.35 \\
\hline unit-2 & -5.21 \\
\hline unit-3 & -5.17 \\
\hline unit-4 & -5.25 \\
\hline unit-5 & -4.89 \\
\hline unit-6 & -5.31 \\
\hline unit-7 & -5.24 \\
\hline MKM99 & -6.6 \\
\hline
\end{tabular}

Tableau 5.1. Curvature in the zone $\mathrm{y}^{+}>\delta_{\mathrm{n}}$ calculated by model-free simulations [3] and a direct simulation [13]

\subsection{Model of the turbulence in discrete mechanics}

One of the fundamental hypotheses of discrete mechanics is that the pressure effects are separable from the viscosity effects. Another key assumption is that the sum of all forces per unit mass can be expressed as two potentials, a scalar potential for the pressure stress and a vector potential for the shear-rotation stress $\gamma=-\nabla \phi^{o}+\nabla \times \boldsymbol{\psi}^{o}$, where $p^{o}=\rho_{v} \phi^{o}$ is the mechanical equilibrium pressure and $\boldsymbol{\omega}^{o}=\rho_{v} \boldsymbol{\psi}^{o}$ is the equilibrium rotation stress. The equilibrium pressure and shear-rotation stresses are accumulators of the pressure and viscosity forces.

Recall the discrete equations established earlier:

$$
\left\{\begin{array}{l}
\frac{\partial \mathbf{V}}{\partial t}+\frac{1}{2} \nabla\left(\|\mathbf{V}\|^{2}\right)-\frac{1}{2} \nabla \times\left(\|\mathbf{V}\|^{2} \mathbf{n}\right)=-\nabla\left(\frac{p^{o}}{\rho_{v}}-d t c_{l}^{2} \nabla \cdot \mathbf{V}\right) \\
-\nabla \times(\nu \nabla \times \mathbf{V})+\mathbf{g} \\
p=p^{o}-d t \rho_{v} c_{l}^{2} \nabla \cdot \mathbf{V} \\
\rho=\rho^{o}-d t \rho_{v} \nabla \cdot \mathbf{V}
\end{array}\right.
$$

where $\rho_{v}$ is the density associated with the inertial or gravitational mass, defined as a constant on the edge $\Gamma$, whereas $\rho$ is the density defined on the vertices of the primal mesh. The system (1) is autonomous and 
does not need to be accompanied by any state equations. Only the physical parameters themselves must be known. It doesn't matter how these parameters are determined: from tables, correlations, databases, or by any other means. These quantities are local and instantaneous and can depend on the variables themselves. After the system has been solved, the quantities $(p, \rho)$ are advected at the velocity of the fluid. This step is best accomplished by a Lagrangian transport calculation. The term in $\|\mathbf{V}\|^{2}$ from Equation (1) can be incorporated into the gradient, corresponding to the dynamic pressure, and associated with the accumulation pressure $p^{o}$ to give the Bernoulli pressure.

The formulation of the system (1) is the most compatible with the original discrete perspective. The underlying idea is that the evolution of the physical system is observed as it passes from one mechanical equilibrium state to another. Interpreting the system in terms of motion in a Galilean frame does not change the equilibria themselves, which are entirely governed by the Lagrangian equations of motion. Note that the system (1) represents every kind of flow, mechanical behavior, and wave, including shock waves. Solving this system by a direct simulation (DNS) gives all of the physical information that are expected - not just the velocity or the displacement fields, but also the instantaneous pressure and shear stresses. In particular, the system represents turbulent flows if the spatial and temporal scales are chosen accordingly.

The spatial scale characterizing a turbulent flow becomes smaller as the Reynolds number of the flow increases. Interactions between different scales of turbulence generate an apparent diffusion that could potentially be used to define a turbulent kinematic viscosity $\nu_{d m}$ in the context of an ad hoc model. Note that the fluid is assumed to be Newtonian and the turbulent dynamic shear viscosity $\mu_{d m}$ is not an intrinsic property but depends on the characteristics of the flow.

This idea of apparent viscosity can be incorporated into the discrete model. Unlike the turbulent viscosity $\mu_{t}$ of a statistical model, the apparent viscosity is not defined in terms of an equilibrium between production and dissipation. The production does not appear anywhere directly; for example, it cannot be found in the Navier-Stokes equations. The viscosity represented by the quantity $\nu_{d m}$ is not an intrinsic property of the fluid, but it is nonetheless a physical quantity. Knowledge of this quantity can be exploited to model the turbulence realistically. The objective of this section is to apply the concepts of discrete mechanics to establish a model of turbulent flows phrased exclusively in terms of the averages of specific quantities such as the velocity or the pressure stress.

\subsection{Application to a turbulent flow in a channel with $\mathbf{R e}_{\tau}=590$}

Historically, one of the most emblematic problems ever studied since the original experiments by Reynolds is the flow within a channel, which becomes turbulent at Reynolds numbers of the order of $R e \approx 2300$. Many experimental studies, Direct Numerical Simulations (DNS), Large-Eddy Simulations (LES), and even Reynolds-Averaged Navier-Stokes Equations (RANSE) statistical models have examined this problem, resulting in extremely accurate databases.

The problem setup is straightforward. A flow with a constant Reynolds number is considered within a channel with rectangular cross-section, driven by a fixed pressure difference $\delta p$ in the $x$-direction. Since the length of the channel is assumed to be large relative to its hydraulic diameter, the average pressure gradient $\nabla p$ is constant along the channel. To simulate this problem, periodic boundary conditions are typically assumed in the $x$ - and $z$-directions. 
Each zone of the flow in the channel is modeled directly from Equation (1). The conclusions of our model will be fully consistent with the original dimensionless analysis that was performed to describe the behavior of this flow. But for now, these equations are already fully representative of any laminar or turbulent flow; the analysis that we shall perform below is based on the equilibrium between the pressure and the curl of the velocity brought by the discrete mechanics equations. In particular, between any two layers of the fluid (1) and (2), the following equality holds:

$$
-\nu_{1}(\nabla \times \mathbf{V})_{1}=-\nu_{2}(\nabla \times \mathbf{V})_{2}
$$

This condition is intrinsically satisfied. It replaces the equality of shear stresses described by the Cauchy tensor. It also holds for two immiscible fluids in problems with multiphase flows.

Since we are only interested in finding the steady average flow, the partial derivative of the velocity with respect to time is zero. The two inertial terms are also zero. This property is of course not satisfied in direct simulations. The equations of motion characterizing established turbulent motion in a channel with constant cross-section may therefore be expressed as follows in terms of average values:

$$
-\nabla p^{o}-\nabla \times\left(\mu_{d m} \nabla \times \mathbf{V}\right)=0
$$

The Hodge-Helmholtz theorem defines the gradient of a scalar function and the curl of a vector quantity as being orthogonal. The two fields $\nabla p$ and $\nabla \times \boldsymbol{\omega}$ are equal and orthogonal, and so must both necessarily be equal to a constant. Since the average pressure field is only a function of $x$ and the shear field is only a function of $y$, Equation (3) could in principle be reduced to partial derivatives along $x$ and $y$. However, we shall avoid this reduction, since these operators have a physical meaning that disappears if we replace $\nabla \times \mathbf{V}$ by $d \mathbf{V} / d y$, which is just part of the component of the curl.

This zone of the flow is defined by the reduced coordinates $y^{+}=y u_{\tau} / \nu<\delta_{v}$, where $u_{\tau}=\sqrt{\tau_{w} / \rho}$ is the shear velocity and $\nu$ is the kinematic viscosity. The reduced velocity is classically defined by $u^{+}=u / u_{\tau}$. The characteristic length $\delta_{v}$ of this zone can thus be determined by studying the turbulent boundary layer of a planar sheet, where it is typically equal to $y^{+} \approx 7$.

Within this layer, the viscous effects are dominant and any remaining velocity is maintained by the outer layers. This is a Couette flow generated by the adjacent fluid, known as the buffer zone. However, in a planar Couette flow, the curl is constant and the pressure gradient is zero. This is not the case for the average gradient $\nabla p^{o}$. The only way to recover Couette flow in this zone is to take $\nabla \times \boldsymbol{\omega}^{o}=\nabla p^{o}$ and $\mu_{d m}=\mu$; Equation (3) then becomes:

$$
\nabla \times \mathbf{V}=0
$$

i.e. $u^{+}\left(y^{+}\right)=\mathbf{V} \cdot \mathbf{e}_{x}=y^{+}$. This recovers the classical linear law, except that the slope is imposed by the external flow.

On a physical point of view, the imposed pressure in any given cross-section is determined solely by the constant gradient $\nabla p^{o}$. This is especially true if the thickness $\delta_{l}$ is very low. Without the term in $\nabla \times \mathbf{V}$, the gradient in the viscous sublayer cannot be eliminated, and the law $u^{+}=f\left(y^{+}\right)+$is not linear but quadratic. Hence, the shear stress is steady and accumulates in $\boldsymbol{\omega}^{o}$ according to the law $\boldsymbol{\omega}^{o}\left(y^{+}\right)=y^{+} \mathbf{e}_{z}$. 
In boundary layer theories, these two regions - the buffer zone and the logarithmic zone - are separate. This is not the case here, in discrete mechanics. The buffer zone is not just a coupling zone but also plays an essential role in the equilibrium between the pressure and the viscous stresses. These two effects are of the same order and modeling them can be particularly tricky. We shall assume that the flow is unsteady in each zone, allowing the accumulated viscous stresses to be destroyed, with $\alpha_{t}=0$. Consequently, the flow is generated by the constant pressure gradient $\nabla p^{o}=\delta$. The equations of motion now become:

$$
-\nabla p^{o}-\nabla \times\left(\mu_{d m} \nabla \times \mathbf{V}\right)=0
$$

which gives:

$$
\frac{d}{d y^{+}}\left(\mu_{d m} \frac{d u^{+}}{d y^{+}}\right)=\delta
$$

Since $\delta$ is a constant, we must have $\mu_{d m}=y^{+2}$, since the product $y^{+} \cdot d u^{+} / d y^{+}$is constant, as established by dimensionless analysis and corroborated by direct simulations.

The solution of the above equations with $\mu_{d m}=y^{+2}$ is:

$$
u^{+}=\delta \operatorname{Ln}\left(y^{+}\right)-\frac{a}{y^{+}}+b
$$

The logarithmic law is recovered as expected, but there is also an additional term in $1 / y^{+}$that increases in significance as $y^{+}$approaches $\delta_{v}$ and becomes negligible as $y^{+}$approaches $\delta_{l} \approx 140$ at the end of the logarithmic zone.

The constants $a$ and $b$ can be deduced from the profiles calculated by DNS, but they are no longer relevant anyway after Equation (5) is integrated directly. The coupling between the different zones is ensured by the implicit condition (2). Hence, the apparent viscosity $\mu_{d m}$ is equal to $\mu$ in the viscous sublayer, increases slowly in the buffer zone, and increases more rapidly in the logarithmic zone. These variations are fully determined by the apparent viscosity in these two zones, $\mu_{d m}=y^{+2}$.

In the kernel of the flow, after $y^{+}=\delta_{l} \approx 140$, the turbulence is fully developed and the diffusion of momentum is maximal; in other words, $\mu_{d m}$ attains its highest values in this zone. In this region, the flow is mainly characterized by the pressure gradient. To satisfy Equation (5), we must invoke the results of direct simulations, as well as the physical fact that this zone has a high mixing rate. The average velocity profiles can be very precisely represented by a second-order law that takes into account the symmetry associated to the axis of the channel. This law can be expressed in the form $u^{+}=u_{m}^{+}-y^{+2} / \kappa$, where $\kappa$ is the absolute value of the curvature of the velocity profile. This is a constant that depends on the turbulent Reynolds number $R e_{\tau}$. This gives the value $\mu_{d m}=-\delta / 2 \kappa=\mu_{m}$ of the viscosity. The apparent viscosity is constant for a given turbulent Reynolds number.

Let us briefly present the results obtained by the discrete approach [1] for the flow in a channel with $R e_{\tau}=590$. Consider a 3D channel with rectangular cross-section whose lateral dimensions in the $x$ and $z$-directions are larger than the vertical dimension in the $y$-direction. No-slip conditions are imposed on the horizontal walls; periodic boundary conditions are applied in the $x$-direction. 
A constant pressure gradient $\delta p \mathbf{e}_{x}$ is maintained throughout the volume to fix the value of the average velocity $V_{0}=\mathbf{V}_{0} \cdot \mathbf{e}_{x}$. The dimensions and the velocity $V_{0}$ are chosen in such a way that the turbulent Reynolds number $R e_{\tau}$ is 590. Dimensionless quantities $\mathrm{y}+$ and $\mathrm{u}+$ are then derived from the vertical size of the channel and the local velocity based on Vo.

This configuration has been extensively studied since the early 1980 s by performing direct simulations or large-eddy simulations. The results are broadly consistent at low Reynolds numbers like these. We can cite the work by Kim, Moin, and Mansour [6] or Moser, Kim, and Mansour [13] for example. More recently, other authors have published refined comparisons that were reviewed in a test case organized by Denaro in 2011. The results were updated in 2014 and are available in the literature [3].

Our objective here is to develop a steady turbulence model for the averaged parameters that is fully based on the discrete-mechanical approach and which may be applied to the discrete equations arising from this model. Even if the Navier-Stokes equations lead to identical results when the physical properties are constant, they differ significantly from the discrete equations, meaning that a new model of turbulence is needed for the discrete approach. The concept of rotation effectively lies at the heart of the discrete theory, which describes viscous effects solely in terms of the vector potential $\boldsymbol{\omega}$ rather than a stress tensor.

Many of the approaches currently used to model the turbulence appear to conflate the diffusion of momentum and the viscous dissipation. These are two different effects, and most models from statistical modeling and LES are in fact based on the dissipation.

In discrete mechanics, the transfer of momentum within the fluid is governed by the dual curl and the shear-rotation stress $\boldsymbol{\omega}$ through the term $\nabla \times\left(\boldsymbol{\omega}^{o}-\mu \nabla \times \mathbf{V}\right)$. The quantity $\boldsymbol{\omega}^{o}$ represents the accumulation stress of the diffusion effects associated with $\mu$, the shear-rotation stress, which is the asymptotic value of the instantaneous effects. For solids or fluids with very small time constants, this term can be written as $d t \mu_{e}$, where $\mu_{e}$ is the elastic shear modulus. In fact, this time constant corresponds to the period $d t$ between two observations of the physical system.

In turbulence, this characteristic timescale becomes smaller as the Reynolds number becomes larger. The shear stresses are diffused by vortices, which creates an apparent diffusion phenomenon as a result of mixing. We can define viscosity arising from this turbulence, but it represents a completely separate concept from the viscous dissipation, which does not appear directly in the equations of motion. It is neither a turbulent viscosity, nor a subgrid-scale viscosity. We shall call this viscosity $\mu_{d m}$, and it will of course depend on the local conditions of the flow.

The simulation was performed in a 2D domain with periodic conditions in $x$ and a mesh with 100 cells in the $x$-direction and 64 cells in the $y$-direction. The initial conditions on $(p, \mathbf{V})$ were set to zero and the pressure gradient was chosen in such a way that $R e_{\tau}=590$. The steady solution was calculated up to the machine error. A one-dimensional model was also developed and the results are identical. The steady version of the problem that is periodic in $x$ effectively reduces to a one-dimensional equation along $y$.

The problem is modeled implicitly by layers using the term $\nabla \times\left(\boldsymbol{\omega}^{o}-\mu_{d m} \nabla \times \mathbf{V}\right)$, which already appears in the equations of motion, based on the various zones of the flow: 
- the laminar boundary sublayer for $y^{+}<\delta_{1}$;

- the buffer zone for $\delta_{1}<y^{+}<\delta_{2}$;

- the logarithmic zone for $\delta_{2}<y^{+}<\delta_{3}$;

- the fully turbulent zone for $y^{+}>\delta_{3}$.

The values of the boundaries $\delta_{1}, \delta_{2}, \delta_{3}$ are defined from values in the literature that are consistent with previously conducted direct simulations. A strictly linear law $u^{+}=f\left(y^{+}\right)$can only be obtained for the accumulation stress $\boldsymbol{\omega}^{o}$, since the constant gradient maintained within the laminar boundary sublayer is not compatible with a linear law. However, the thickness $\delta_{1}$ is very small and the pressure is the same as in the buffer zone. In practice, the term $\boldsymbol{\omega}^{o}$ balances out the effect of the pressure gradient and the velocity law is perfectly linear in the boundary sublayer.

The physical explanation for this is as follows: within the laminar boundary sublayer, the flow is steady, and $\mu$ has attained its asymptotic limit, known as the molecular viscosity. After this point, unsteady exchanges between vortices cause a small part of the rotation stresses to accumulate, but anything that accumulates is then immediately diffused by these same exchanges. This leads to larger viscosity values, $\mu_{d m}>\mu$.
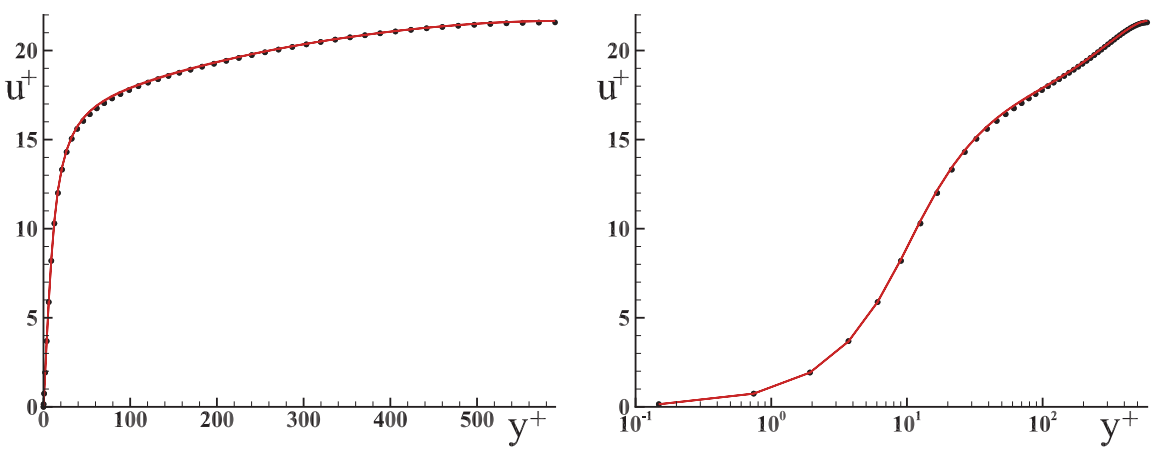

Figure 7. Turbulent channel with $\operatorname{Re}_{\tau}=590$, comparison between the results of DNS (unit-2) [3] (solid line) and the discrete approach [1] (dots). The profile $\mathrm{u}^{+}=\mathrm{f}\left(\mathrm{y}^{+}\right)$of the average velocity is shown in reduced coordinates on the left and logarithmic coordinates on the right.

Figure 7 shows the average velocity $u^{+}=f(y)$ in physical coordinates and $u^{+}=f\left(y^{+}\right)$in logarithmic coordinates. The distinct zones of the flow are clearly distinguishable. The results are compared against the results found by Germano, reproduced from [3]. The objective of this section is to use physical concepts to establish a robust and reliable averaged model with as few parameters as possible that can be determined from direct simulations. Figure 8 plots the values of $u^{+}\left(y^{+}\right)$over time obtained by the discrete model against the results of direct simulations recorded in the reference [3]. The points issued to the model accurately reproduce the evolution in dimensionless coordinates. The dispersion of the results of the direct simulations can be attributed to methodological differences in the authors' approaches, as well as refinements of the mesh near the walls.

Figure 9 gathers together the results for the reduced velocity $\mu_{d m} / \mu$ as a function of the reduced position $y^{+}$in physical coordinates and logarithmic coordinates. In the central zone, the ratio is essentially constant. The same is true in the viscous boundary sublayer, where the ratio is equal to one. Between these two zones, the evolution is quadratic. 


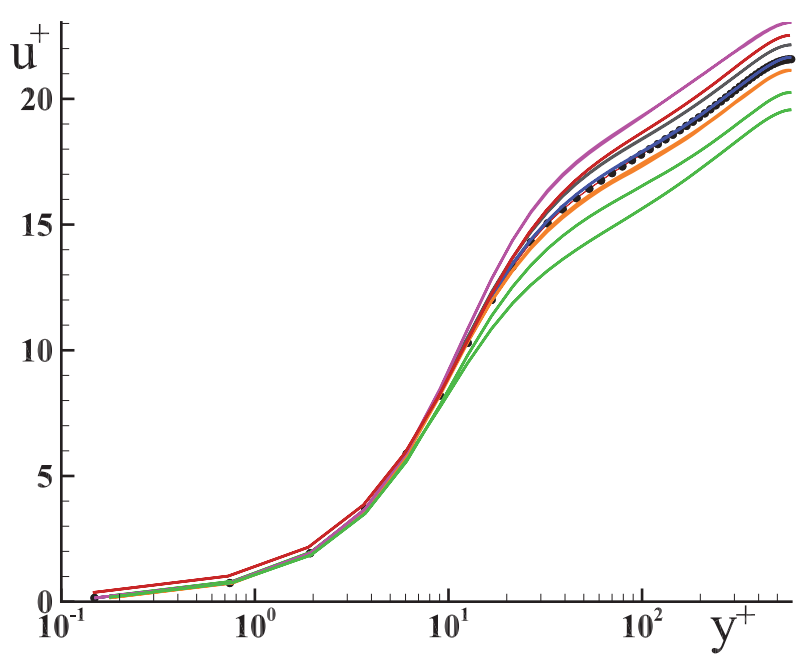

Figure 8. Turbulent channel with $\operatorname{Re}_{\tau}=590$. Results of the TDM model (dots) compared to direct simulations reproduced from [3] (solid lines).

The simulations by Moser et al. [13] are one of the possible references for this turbulent flow problem in the literature. Figure 10 plots the reduced velocity as a function of the reduced position in wall units, showing that these reference results are highly consistent with the results of the discrete model.
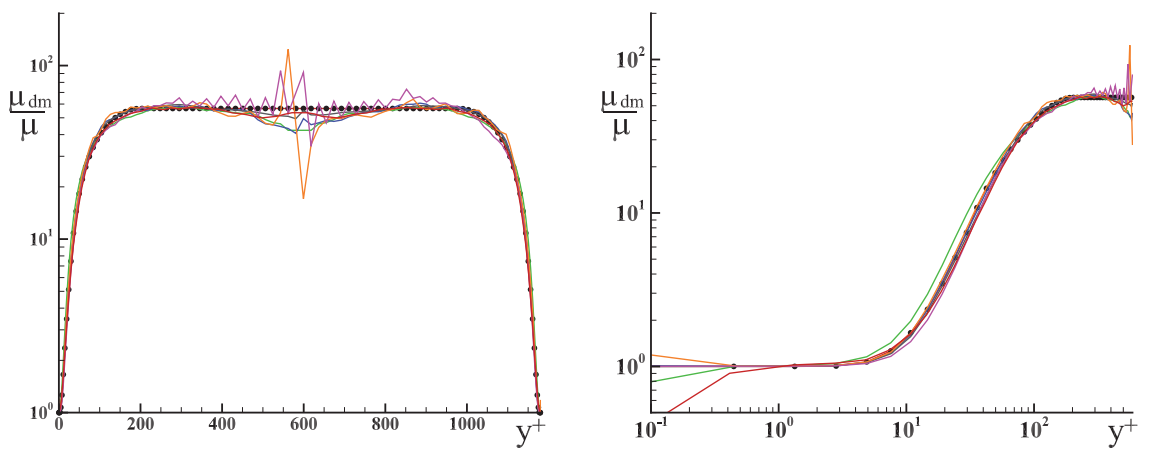

Figure 9. Turbulent channel with $\operatorname{Re}_{\tau}=590$. The reduced turbulent viscosity $\mu_{\mathrm{dm}} / \mu$ is shown for the TDM model (dots) and the DNS by [3] (solid lines).
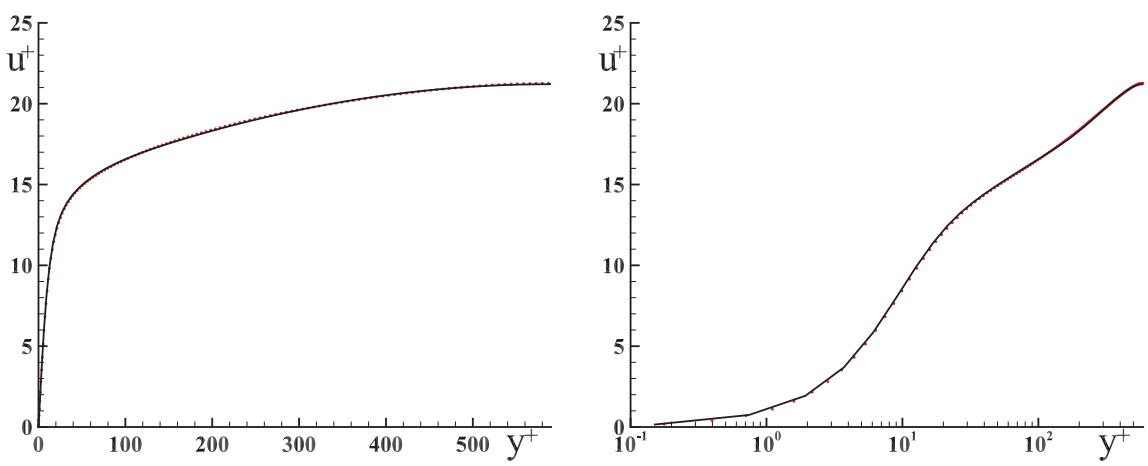

Figure 10. Turbulent channel with $\operatorname{Re}_{\tau}=590$, results from MKM99 [13] (solid line) and from the TDM model (dots). The average velocity profile $\mathrm{u}^{+}=\mathrm{f}\left(\mathrm{y}^{+}\right)$is shown in wall units on the left and in logarithmic coordinates on the right.

We do not impose or even consider any laws on the velocity profiles, since they can be implicitly reproduced by choosing the viscosity $\mu_{d m}$ accordingly. So, which parameters do we need to model the 
flow in a turbulent channel, and hence to model an arbitrary problem? In the viscous boundary sublayer, the viscosity is given by $\mu$, the molecular viscosity, but the thickness $\delta_{v}$ of this layer needs to be known. The intermediate layer requires us to know another thickness to determine the end of the logarithmic zone, and the turbulence zone presented above requires us to know $\kappa$, the curvature of the velocity profile in this zone. Therefore, we at the very least need the three parameters $\delta_{v}, \delta_{l}$, and $\delta_{n}$, as well as $\kappa$ itself. This model also needs to satisfy various constraints. In particular, it must naturally reduce to Poiseuille's solution for a laminar flow in the degenerate case. In this case, the curvature $\kappa$ is given by $\kappa=-1 / 2 \mu$, and the viscous sublayer and the intermediate zone vanish. The laminar flow occupies the whole channel.

Locally, the viscosity $\mu_{d m}$ can be calculated from the equilibrium between the pressure effects and the shear effects as follows in discrete mechanics:

$$
\mu_{d m}=\frac{\nabla p}{\nabla \times \nabla \times \mathbf{V}}
$$

where the denominator is implicitly understood as $\nabla_{d} \times \nabla_{p} \times \mathrm{V}$. The term $\nabla_{d} \times$ is the dual curl and $\nabla_{p} \times$ is the primal curl. This is in fact just the curvature of the velocity profile.

The two vector quantities in Equation (8) both have the same direction as the unit vector $\mathbf{t}$ of the edge $\Gamma$. It therefore makes sense to define the scalar $\mu_{d m}$ on this same edge. Statistical models produce much more fragmented results involving the introduction of so-called universal constants. Furthermore, models based on Boussinesq's hypothesis and the concept of turbulent viscosity $\mu_{t}$ typically only hold in specific conditions - high Reynolds numbers, low Reynolds numbers, etc.

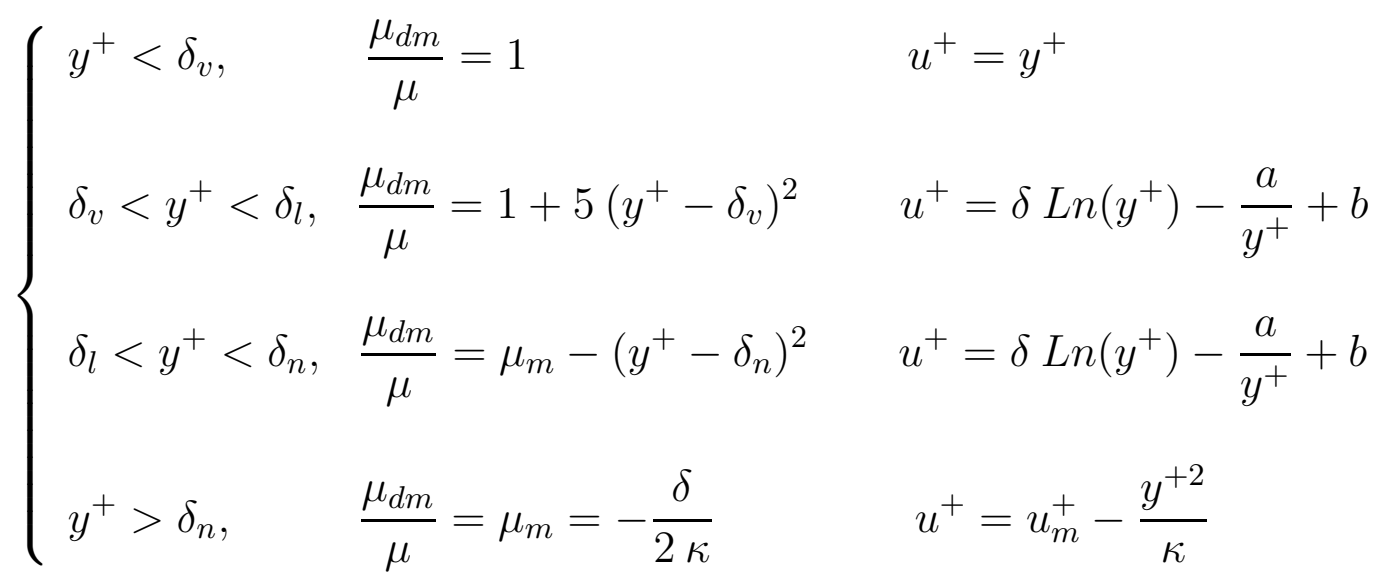

The system (9) summarizes the law of the wall in each zone as a function of the reduced position. This zones are defined by the distances $\delta_{v}=2, \delta_{l}=35, \delta_{n}=200$, and $\mu_{m}=-\delta / 2 \kappa$.

\section{Conclusions and future works}

Discrete mechanics have been rigorously compared and differentiated to Navier-Stokes equations first based on theoretical and modeling issues related to turbulent pressure, vorticity in two-dimensions, divergence of Lamb vector or dissipation. On theoretical aspects some conclusions can be drawn. First, in DNS with Navier-Stokes equations, the pressure fluctuations propagate at celerity $c_{l}$, i.e. $c_{l}=\infty$ for incompressible flows. In any case, incompressible flows or not, the use of a state equation does not account for instantaneous pressure fluctuations. 
By considering a simple rotational flow without deformation, it was demonstrated that it is impossible to express the Lamb vector as a curl in classical mechanics. This form of inertia introduces a term $I_{2}$, the second invariant of gradient velocity tensor, appears when the divergence operator is applied to the acceleration vector. With this form, while the divergence of the velocity is zero, the divergence of the acceleration is different from zero. For example, for a divergence free stationary two-dimensional flow, the term $I_{2}$ is applied to the orthogonal component of the plane considered. Moreover, the term $I_{2}$ is found in the expression of the viscous dissipation associated with the Navier-Stokes equation. All these difficulties are inherent to the notion of continuous medium and in particular to that of the derivation of a vector. These artefacts do not exist in a discrete medium, all the terms of the equation of motion such as the divergence of the acceleration or the viscous dissipation are expressed in a coherent way with the proposed concept.

In a final section, the simulation of the turbulent channel flow at $R e_{\tau}=590$ has demonstrated that the formulation of acceleration equation brought by discrete mechanics, which is intrinsically different from the momentum equations in Navier-Stokes model, allows to recover accurately the DNS and LES results of the literature $[13,3]$. A clear evidence of the physical meaning of DM for turbulence flow modeling was given. In addition, based on DM, the fitting of simulation results was possible for the mean streamwise velocity without any requirement of dimensional analysis considerations. The obtained law is valid from the wall to the center of the channel with a nice agreement to DNS reference values.

Future works and extension of DM use for turbulent motions are oriented in two directions: analysis of well known turbulent flows such as Homogeneous Isotropic Turbulence (HIT), two-phase flows, jets, ... on one hand and building of new LES or RANS modeling based on the new DM formulation of motions on the other hand.

\section{Acknowledgments}

The authors wish to thank Filippo Denaro for providing data on comparative tests on turbulent channel flow at $\operatorname{Re}=590$.

This work was granted access to the HPC resources of IDRIS, CCRT and CINES under the allocation A0032b06115 founded by GENCI (Grand Equipement National de Calcul Intensif).

\section{Bibliography}

[1] J-P. Caltagirone. Discrete Mechanics, concepts and applications. ISTE, John Wiley \& Sons, London, 2019.

[2] J. Deardorff. A numerical study of three-dimensional turbulent channel flow at large reynolds numbers. J. Fluid Mech., 41:453-480, 1970.

[3] F.M. Denaro and al. A comparative test for assessing the performances of large-eddy simulation codes. Proc. XX AIMETA, Bologna, 2011.

[4] C.W. Hamman, J.C. Klewick, and Kirby R.M. On the Lamb vector divergence in Navier-Stokes flows. J. Fluid Mech., 610:261-284, 2008.

[5] F.H. Harlow and J.E. Welch. Numerical calculation of time-dependent viscous incompressible flow of fluid with a free surface. Physics of Fluids, 8:2182-2189, 1965. 
[6] J. Kim, P. Moin, and R. D. Moser. Turbulence statistics in fully developed channel flow at low reynolds number. $J$. Fluid Mech., 177:157-159, 1987.

[7] A.N. Kolmogorov. The local structure of turbulence in incompressible viscous fluid for very large reynolds numbers. Proc. R. S. London, 434:9-13, 1991.

[8] A. Kozachok. Navier-Stokes first exact transformation. Universal Journal of Applied Mathematics, DOI: 10.13189/ujam.2013.010301, 1:157-159, 2013.

[9] H. Lamb. Hydrodynamics. 6 e edition, Dover, New-York, 1993.

[10] J. Lindgren. On the Lamb vector divergence, evolution of pressure fields and Navier-Stokes regularity. arXiv:1206.1281v3, 2012.

[11] J.L. Lumley. Some comments on turbulence. Phys. Fluids, A4:203-211, 1997.

[12] H. Marmanis. Analogy between the Navier-Stokes equations and Maxwell's equations: application to turbulence. Phys. Fluids, 10(6):1428-1437, 1998.

[13] R. D. Moser, J. Kim, and N. N. Mansour. Direct numerical simulation of turbulent channel flow up to $R e_{\tau}=590$. Phys. Fluids, 11:943-945, 1999.

[14] I. Newton. Principes Mathématiques de la Philosophie Naturelle traduit en francais moderne d'après l'œuvre de la marquise Du Châtelet sur les Principia. fac-similé de l'édition de 1759 publié aux Editions Jacques Gabay en 1990, Paris, 1990.

[15] S.B. Pope. Turbulent flows. Cambridge University Press, Cambridge UK, 2000.

[16] O. Reynolds. An experimental investigation of the circumstances which determine whether the motion of water shall be direct or sinuous, and the law of resistance in parallel channels. Proc. R. Soc. London, 35:84-99, 1883.

[17] P. Sagaut. Large eddy simulation for incompressible flow - an introduction. Springer-Verlag, 2001.

[18] T. Von Kàrmàn. Progress in the statistical theory of turbulence. Proc. National Academy Sci. United States America, 1948. 